\title{
EDITORIAL
}

\section{Preface to the second special issue on Terahertz Wave Science, Technology, and Application}

\author{
Jianquan YAO $(\bowtie)^{1,2}$ \\ 1 The Institute of Laser \& Optoelectronics, Key Laboratory of Opto-electronics Information Technology (Tianjin University), \\ Ministry of Education, Tianjin 300072, China \\ 2 Wuhan National Laboratory for Optoelectronics, Huazhong University of Science and Technology, Wuhan 430074, China
}

(C) Higher Education Press and Springer-Verlag Berlin Heidelberg 2015

Terahertz $(\mathrm{THz})$ frequency region has attracted considerable interest, due to its unique nature and promising applications. A number of breakthroughs were achieved by the worldwide research groups in this field, including high-performed sources, detectors, functional devices, interaction with materials as well as applications. Previously, the first special issue on "Terahertz Wave Science, Technology, and Application" was presented (June 2014). This is the second special Issue with 3 review articles and 8 research articles, which introduce and discuss recent developments of relevant subjects.

These papers can be divided into such categories: nonlinear optical response of grapheme, semiconductor activated metamaterials, superconducting metamaterials, $\mathrm{THz}$ generation with air and GaAs photoconductive antenna, double-probe-pulse $\mathrm{THz}$ detection, applications in sensing, imaging and spectroscopy, and so on.

Prof. Chao Zhang and coworkers of University of Wollongong reviewed the recent developments of nonlinear optical response of graphene in $\mathrm{THz}$ and near-infrared frequency regime. Prof. Hou-Tong Chen of Los Alamos National Laboratory reviewed the recent progresses on semiconductor activated THz metamaterials. Prof. Weili Zhang and coworkers of Tianjin University reviewed the recent results on tailoring electromagnetic responses in $\mathrm{THz}$ superconducting metamaterials.

In the research articles, Prof. Kun Zhao's group of China University of Petroleum reported the detection of nheptane, n-octane and nitrate solutions with THz time-domain spectroscopy. Prof. Zhi Hong and coworkers of China Jiliang University investigated a high $Q \mathrm{THz}$ asymmetrically coupled resonator and its sensing performance. Prof. Alexander Shkurinov's group of Lomonosov Moscow State University demonstrated the simultaneous generation of nonlinear optical harmonics and $\mathrm{THz}$ radiation in air: polarization discrimination of various nonlinear contributions. Prof. J. Bianca Jackson and coworkers of Ecole Polytechnique applied $\mathrm{THz}$ pulse imaging in archaeology. Dr. Haiwei Du of RIKEN investigated optical ionization evolution effect on photocurrent produced from two-color femtosecond laser pulses. Prof. Jinsong Liu's group presented the studies on the behavior of GaAs photoconductive antenna.

The articles in this special issue have certain significance for promoting the development of $\mathrm{THz}$ technology. It is hoped that the second special issue will benefit THz applications.

On behalf of the editorial committee, we express our sincere thanks to all the authors and reviewers for their great contribution to this special issue. Also, we thank the editorial committee members for their excellent assistance. Finally, we are grateful to the editorial staff for their help, also thanks for my research assistants Dr. Pengxiang Liu, Dr. Lanju Liang and Yating Zhang. Without all of the contributions of these hard-working, dedicated people, it would have been impossible to produce this second special issue.

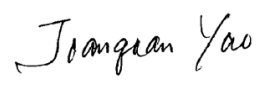

Received January 16, 2015

E-mail: jqyao@tju.edu.cn 


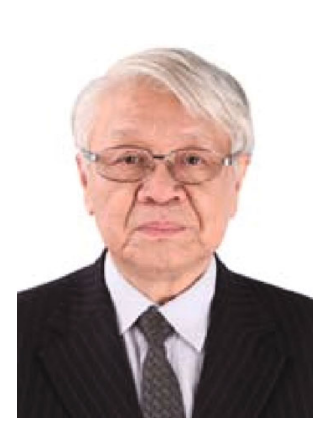

Jianquan Yao was born in Shanghai, China, in 1939. He graduated from School of Precision Instrument Department of Tianjin University in 1965.

Since 1966, he joined Tianjin University as an Assistant Professor and Lecture. Now, he is a Professor and Director of The Institute of Laser \& Optoelectronics of Tianjin University. As a visiting scholar, he joined the Department of Applied Physics, Stanford University, USA, from 1980 to 1982 . By invitation, he visited Princeton University, University of Pennsylvania, University of Southern California (USC), St. Andrews University and some university in USA, UK, France, Germany, Russian, etc. He was an adjunct professor of The Hong Kong University of Science and Technology (HKUST). He has long been engaged in the research of all-solid laser, nonlinear optics frequency conversion and terahertz science \& technology. His theory of precise calculation of optimum phase matching of biaxial crystal has been called the "Yao and Fahlen Technology". He advanced "Gaussian like distribution" of laser resonant theory. He developed the theory of laser frequency doubling under high conversion efficiency and new method with quasi-continuous-wave (quasi-CW) pumping. He developed an intracavity frequency doubled Nd:YAG laser whose output power was up to $100 \mathrm{~W}$, and this laser source was used to establish laser three dimension scanning photography system. He invented the dye and titanium sapphire tunable laser systems pumped by quasi-CW green laser source. He established a quasi-CW pumped-tunable laser technical system, making important contributions to the development of the new solid-state laser and tunable laser technologies.

Recently, he was focusing his research work to terahertz wave generation by difference frequency generation (DFG) and terahertz parametric oscillator (TPO) and some method with optical, nonlinear optics and applications.

Prof. Yao has been awarded World Gold Medal of Invention, Eureka, Brussels, and National Invention Award Class II of China. He has been awarded Second Prize for Chinese Ministry of Education and Tianjin City Invention, etc. He has published over 850 papers. Three books: "Nonlinear Optical Frequency Conversion and Tunable Laser Technology" (Science Press, 1995 in China), "All Solid State Laser and Nonlinear Optical Frequency Conversion Technology" (Science Press, 2007 in China) and "Nonlinear Optics and Solid-State Laser, Advanced Concepts, Tuning-Fundamentals and Applications"(Springer, 2012 in USA) were the summary of his research achievements. Under his instruction, 18 post Ph.D. students, 112 Ph.D. students and 150 master degree students have obtained their degrees.

In 1997, Prof. Yao was elected as an Academician of Chinese Academy of Sciences. 